\title{
Allergic bronchopulmonary aspergillosis in a patient with chronic obstructive pulmonary disease
}

\author{
Elias Mira,*Ashok Shaha
}

a Department of Respiratory Medicine, Vallabhbhai Patel Chest Institute, University of Delhi, Delhi, India

Originally received 13th April 2011; resubmitted 27th July 2011; revised 16th September 2011; accepted 17th September 2011; online 5th January 2012

\begin{abstract}
Summary
Allergic bronchopulmonary aspergillosis (ABPA) is a debilitating lung disease which occurs as a result of interplay between a variety of host and environmental factors. It occurs in certain susceptible individuals who develop hypersensensitivity to the colonised Aspergillus species. ABPA is a complicating factor in $2 \%$ of patients with asthma and is also seen in patients with cystic fibrosis. Asthma and chronic obstructive pulmonary disease (COPD) are known to share key elements of pathogenesis. It is well known that ABPA can occur in patients with asthma, but it has recently been reported in patients with COPD as well. We report a 55-year-old male ex-smoker who presented with complaints of exertional breathlessness and productive cough for five years and an episode of haemoptysis four days prior to presentation. Spirometery showed aiflow obstruction which was not reversible with bronchodilators. Chest CT scan revealed paraseptal emphysema along with central bronchiectasis (CB) in the right upper lobe and bilateral lower lobes. A type I skin hypersensitivity reaction to Aspergillus species was elicited. He fulfilled the serological criteria for ABPA and was diagnosed as having concomitant COPD and ABPA-CB. The patient was initiated on therapy for COPD along with oral corticosteroids, on which he had remarkable symptomatic improvement.

(c) 2012 Primary Care Respiratory Society UK. All rights reserved.

E Mir and A Shah. Prim Care Respir J 2012; 21(1): 111-114

http://dx.doi.org/10.4104/pcrj.2012.00001
\end{abstract}

Keyw ords allergic bronchopulmonary aspergillosis, central bronchiectasis, chronic obstructive pulmonary disease

\section{Introduction}

Although allergic bronchopulmonary aspergillosis (ABPA) is predominantly a disease of patients with asthma, it is also seen in cystic fibrosis." Impaired mucocilliary clearance and viscid sputum in asthmatic airways favours germination of Aspergillus spores. ABPA is an immunologically-mediated lung disease caused by hypersensitivity in a susceptible individual to the presence of Aspergillus hyphae which colonise the airways. ${ }^{2}$ Chronic obstructive pulmonary disease (COPD) is a debilitating disorder recognised less than two centuries ago by Laennec who described it as "bronchodilation and emphysema". ${ }^{3}$ Though largely seen in smokers, it is now recognised that subjects who are exposed to biomass fuel smoke are also at risk of developing COPD. ${ }^{4}$ Among common diseases afflicting the human population, COPD is the only disorder which is still increasing in prevalence and it is expected to be the third most common cause of death by 2020. ${ }^{5}$ COPD and asthma are believed to share at least some aspects of pathogenesis. ${ }^{6}$ Surprisingly, the presence of ABPA in COPD is rather unusual, the reasons for which remain largely unknown. Recently, two reports have documented the association of Aspergillus sensitisation and the presence of ABPA in patients with COPD. ${ }^{7.8}$ This is another case of a 55-year-old male patient diagnosed as having concomitant COPD and ABPA.

\section{Case history}

A 55-year-old non-atopic male, HIV negative and an ex-smoker, was referred for evaluation of a 5 -year history of progressive pulmonary symptoms. His clinical course was characterised by exertional breathlessness, occasional wheezing, and cough with thick mucoid sputum. He had seasonal aggravation of his symptoms, when he produced about a cupful of non-foul smelling yellow sputum daily. An episode of haemoptysis four days before had prompted the referral. Two years prior to presentation, based on his clinical and radiological profile, he had received antituberculous therapy for nine months (despite sputum samples being consistently negative for acid fast bacilli) with no therapeutic benefit. There were no other co-morbidities.

\footnotetext{
* Corresponding author: Professor Ashok Shah, Department of Respiratory Medicine, Vallabhbhai Patel Chest Institute, University of Delhi, Delhi, 110007, India. Tel: +91-11-25433783 E-mail: ashokshah99@yahoo.com
} 


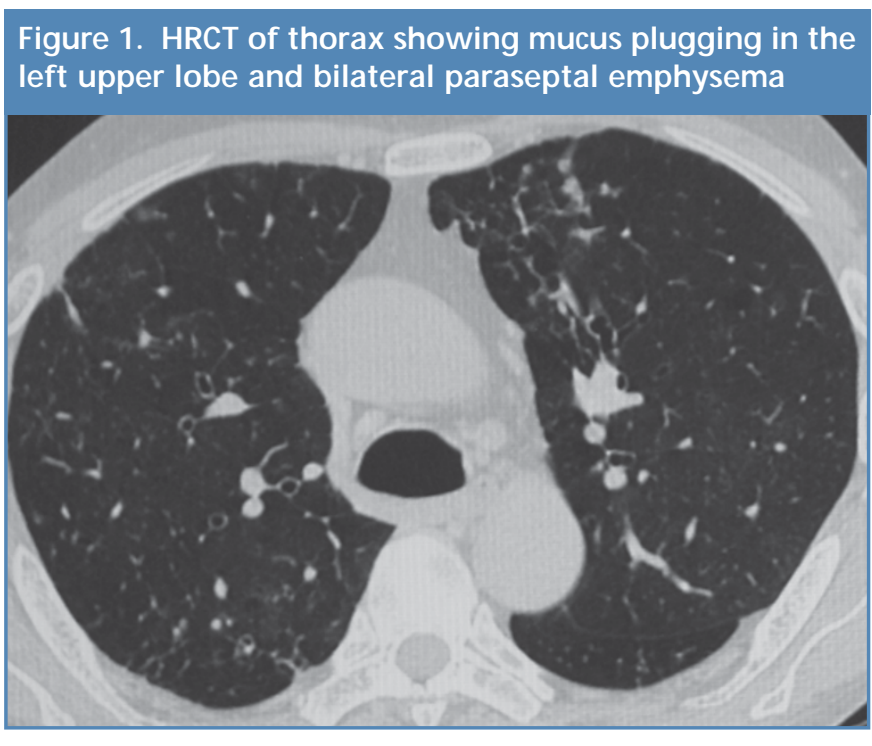

He had smoked 20 cigarettes daily for a period of 30 years (30 pack-years) but had quit five years previously due to an increase in cough and breathlessness. There was no significant family history.

Although the patient had no acute distress, his arterial $\mathrm{SPO}_{2}$ at room air, was $93 \%$. There was no cyanosis or clubbing, and diaphragmatic excursion was equal on both sides. On auscultation, he had prolonged expiration with bibasal coarse crepitations predominantly on the right side. The haemoglobin level was $14.9 \mathrm{gm} / \mathrm{dl}$, along with a total leucocyte count of 14,580 cells/cu-mm with a differential count of neutrophils $73 \%$, lymphocytes 23\%, monocytes 3.0\% and eosinophils $1.0 \%$. Absolute eosinophil count was 240 cells/cu-mm. Several sputum stains and cultures for Mycobacterium tuberculosis as well as other aerobic organisms were negative. A single sputum sample was negative for pathogenic fungi. On pulmonary function testing, FVC was $2.96 \mathrm{~L}$ (93\% of predicted), FEV 1 was $1.81 \mathrm{~L}\left(69 \%\right.$ of predicted), $\mathrm{FEV}_{1} / \mathrm{FVC}$ ratio was 0.61 , FEV $0.5 / F V_{0.5}$ was 0.35 . The total lung capacity was $4.11 \mathrm{~L}(82 \%$ of predicted), residual volume was $1.13 \mathrm{~L}$ (63\% of predicted), RV/TLC was $27 \%$ ( $87 \%$ of predicted) and DLCO was 10.81 $\mathrm{mL} / \mathrm{min} / \mathrm{mmHg}$ ( $45 \%$ of predicted) and diffusion per unit of alveolar volume was $2.76 / \mathrm{min} / \mathrm{mmHg}$ (62\% of predicted). After inhalation of $400 \mu \mathrm{g}$ of salbutamol, the FVC was $3.16 \mathrm{~L}(99 \%$ of the predicted) and $\mathrm{FEV}_{1}$ was $1.96 \mathrm{~L}$ (75\% of predicted). There was no significant reversibility with bronchodilators. This was indicative of moderate airflow limitation (stage II COPD) ${ }^{9}$ with moderately impaired diffusion capacity.

Chest radiograph, done on presentation, showed hyperinflated lungs associated with cystic areas in the right lower zone near the hilum. There were no previous chest radiographs for comparison. The history of haemoptysis prompted a highresolution CT (HRCT) scan of the thorax, which revealed bilateral paraseptal emphysema along with mucoid impaction in the left upper lobe (see Figure 1). Central bronchiectasis as evidenced by
Figure 2. HRCT of thorax showing central bronchiectasis (string of pearls appearance) in the right upper lobe

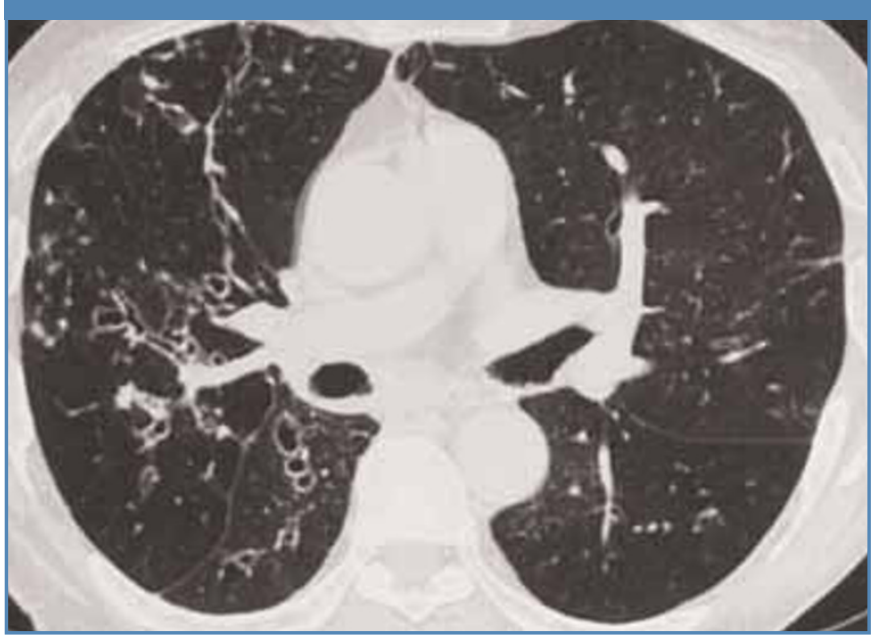

the presence of the "string of pearls" appearance in the inner two thirds along with peribronchial thickening was visualised in the right upper lobe (see Figure 2) as well as both the lower lobes. The presence of central/proximal bronchiectasis, a feature pathognomic of ABPA, raised the suspicion of ABPA which was further strengthened by the detection of mucoid impaction in the left upper lobe. Skin prick test against Aspergillus species was negative; however, intradermal tests against Aspergillus fumigatus and $A$. flavus were positive for type 1 hypersensitivity. Serum precipitins against both these species were positive. Specific lgG against $A$. fumigatus was also positive. Total serum lgE was $645.70 \mathrm{IU} / \mathrm{ml}$ as against a normal value of 0 to $14 \mathrm{lU} / \mathrm{ml}$.

The diagnosis of ABPA was based on: a) type I hypersensitivity to intradermal challenge with extracts of $A$. fumigatus and $A$. flavus; b) elevated total serum IgE levels; $c$ ) presence of serum precipitins against $A$. fumigatus and $A$. Flavus; d) presence of specific lgG against $A$. fumigatus; and e) central/proximal bronchiectasis on the HRCT scan of the thorax. A diagnosis of COPD stage II with allergic bronchopulmonary aspergillosis and central bronchiectasis (ABPA-CB) was made. ${ }^{1,9}$

The patient was initiated on therapy for COPD as per GOLD guidelines. ${ }^{9}$ In addition, oral prednisolone $(0.5 \mathrm{mg}$ per $\mathrm{kg}$ body weight) was commenced which was tapered after 15 days to an alternate day regimen. This was further reduced at the rate of $5 \mathrm{mg}$ per month after three months. Within a fortnight the patient experienced remarkable relief; his cough and breathlessness decreased, while wheezing cleared completely. Though sputum production persisted, it had considerably reduced and was no longer regarded as bothersome. There were no further episodes of haemoptysis. The patient could walk for a longer distance without getting symptoms. The arterial $\mathrm{SPO}_{2}$ at room air increased to $96 \%$ three months later. As he was from a small town 300 kilometres away, after six months the patient expressed his wish not to have regular follow-up. 


\section{Discussion}

ABPA complicates only $2 \%$ of patients with asthma. ${ }^{.}$Although, the pathogenesis of ABPA is largely unknown, a complex interplay of host and environmental factors is thought to be responsible. In some asthma subjects, host factors allow colonisation of Aspergillus species in the viscid mucus. ABPA results from a primarily CD4+ Th2 lymphocyte-mediated immune response to these colonised Aspergillus hyphae in certain susceptible individuals. Direct tissue injury to the airway epithelium caused by fungal proteases also augments the immune-mediated damage to the lung parenchyma. ${ }^{.}$Certain alleles of major histocompatibility complex (MHC) genes like HLA-DR2 or HLA-DR5 ${ }^{10}$, cystic fibrosis transmembrane receptor (CFTR) genes ${ }^{11}$, interleukin (IL) $10^{12}$ and surfactant proteins and mannose binding lectins ${ }^{13}$ seem to predispose an individual to the development of ABPA, while others like MHC DQ2 have been found to be protective. ${ }^{14}$

It has been suggested that COPD and asthma have similar origins and the development of the disease was based upon modulation of host factors by certain environmental variables including smoking. This came to be known as the Dutch hypothesis. ${ }^{15}$ Smokers have been shown to have greater bronchial hyperreactivity, ${ }^{16}$ raised serum $\operatorname{lgE}$ levels, ${ }^{17}$ and peripheral blood eosinophilia, ${ }_{18}^{18}$ than non-smokers. Eosinophilic inflammation of the airways which was reversible with oral corticosteroid therapy has also been demonstrated in a subset of patients with COPD. ${ }^{19,20}$ Similarly neutrophilic inflammation has been shown in some patients with severe asthma. ${ }^{21}$ There are great similarities between the airway inflammation in COPD and severe asthma. ${ }^{9}$ All these observations suggest an overlap syndrome between COPD and asthma, with the two diseases being considered as two ends of a spectrum - the 'asthma' end characterised by minimal parenchymal injury of the lung and normal pulmonary compliance, and the 'COPD' end showing extensive parenchymal injury, alveolar destruction and increased pulmonary compliance. ${ }^{6}$

In COPD, noxious stimuli cause airway inflammation leading to squamous metaplasia which results in impaired mucocilliary clearance. There is also mucous hypersecretion due to the increased number of goblet cells and enlarged submucous glands. ${ }^{22}$ This environment of mucous hypersecretion and impaired mucocilliary clearance is thought to favour colonisation of Aspergillus species as in patients with asthma. Aspergillus colonisation in patients with COPD has been described previously ${ }^{23}$ and it is one of the most common non-malignant diseases associated with invasive aspergillosis in patients admitted to an intensive care unit. ${ }^{24}$

Given these similarities between the pathogenesis of asthma and COPD, the rarity of the association of ABPA and COPD is rather surprising. It remains to be seen whether this is simply an under-recognition of this association or whether patients with COPD are definitely at lower risk of developing ABPA than patients with asthma. Aspergillus sensitisation occurs in 16 to
$38 \%$ of asthma patients across the world. ${ }^{25}$ We have previously reported a positive skin test for hypersensitivity to Aspergillus antigens in $28.5 \%$ of asthma patients. Furthermore, these patients have a more severe form of asthma compared to those who do not demonstrate skin reactivity to Aspergillus. ${ }^{25}$ Of 200 patients with COPD, 17 (8.5\%) had Aspergillus hypersensitivity as compared to none in the control group and only two $(1 \%)$ had serological evidence suggestive of ABPA. ${ }^{8}$ However, neither of the patients had central bronchiectasis nor did they require specific treatment. We evaluated 41 patients with COPD and found that 27 had upper respiratory tract symptoms; of these 27 subjects, 11 had positive skin prick tests but none demonstrated hypersensitivity to Aspergillus species. ${ }^{26}$

The presence of central bronchiectasis and mucus plugging on the HRCT scan of the thorax in our patient were the crucial clues for the diagnosis of ABPA. In India, haemoptysis (as seen in our patient) is not an uncommon feature as it has been documented in up to $41 \%$ of patients with ABPA. ${ }^{27}$ A positive skin prick test is a criterion for the diagnosis of ABPA as it suggests an immediate IgE response. However, it signifies sensitisation to Aspergillus and is not specific for ABPA. It has also been suggested that intradermal skin tests are more sensitive than skin prick tests. 'In a study from India, skin prick tests against Aspergillus were negative in $16(18 \%)$ out of 89 patients of asthma who were diagnosed as ABPA. ${ }^{28}$ Our patient too had a negative skin prick test but a positive intradermal reaction against $A$ fumigatus and $A$ flavus. Eosinophilia is usually present in patients with ABPA, but is not included in the minimal essential criteria. ${ }^{29}$ Although, elevated total serum IgE level is an important criterion for diagnosing ABPA, the levels are known to fluctuate during the course of the disease. ${ }^{29}$ The presence of a negative skin prick test, moderately elevated IgE levels and almost normal eosinophil counts in our patient may represent a quiescent stage of ABPA. It is also possible that ABPA developing in patients with COPD may have a different presentation compared to the ABPA in patients with asthma.

The features suggestive of COPD in our patient include a 30 pack-year history of smoking with progressive exertional breathlessness for the previous five years, together with documented moderate airflow obstruction which was not reversible with bronchodilators. Severely reduced $\mathrm{FEV}_{0.5} / \mathrm{FIV}_{0.5}$ was also observed. ${ }^{30}$ Although TLC and RV/TLC were within normal limits, impaired diffusion capacity was suggestive of COPD. This was further confirmed by the detection of paraseptal emphysema on the HRCT scan, a method more sensitive for detecting emphysema than pulmonary function testing (PFT): ${ }^{31}$ as much as a third of the lungs may be damaged by emphysema before it becomes evident on PFT. ${ }^{31}$ The diagnosis of asthma was unlikely in our patient as he did not have an atopic background or a history of remissions. Furthermore, his symptoms commenced only five years prior to his presentation at the age of 50 .

The difference in the prevalence of Aspergillus 


\section{Learning points for dinicians}

- Allergic bronchopulmonary aspergillosis, ABPA, is a disease of the lungs

- It is known to be a complicating factor in $2 \%$ of patients with asthma and can also occur in patients with cystic fibrosis

- ABPA is caused by hypersensitivity to Aspergillus, usually Aspergillus fumigatus.

- The presence of central bronchiectasis with normal tapering peripheral bronchi is pathognomic of ABPA.

- COPD is usually seen in smokers but can also occur in those chronically exposed to biomass fuel smoke.

- It is thought that asthma and COPD share some aspects of pathogenesis

- The occurrence of ABPA in patients with COPD is unusual

- It is possible that ABPA developing in patients with COPD may present with a slightly different clinical profile as compared to patients with asthma.

hypersensitivity and ABPA in asthma and COPD could possibly be due to some host factors. These include differences in the nature of airway inflammation in COPD and asthma and also the differences in the genetic predisposition of individuals with these two diseases. The frequency of this association needs to be assessed and the reasons for the differences in the prevalence of ABPA in patients with asthma and COPD should be explored.

Conflicts of interest The authors declare that they have no conflicts of interest in relation to this article.

Contributorship EM and AS collected the clinical data and reviewed the literature. EM and AS drafted the manuscript and were responsible for the clinical workup of the patient. AS is responsible for the genuineness of the data and the concept. AS is also the guarantor of the paper.

\section{References}

1. Patterson K, Strek ME. Allergic bronchopulmonary aspergillosis. Proc Am Thorac Soc 2010;7:237-44. http://dx.doi.org/10.1513/pats.200908-086AL

2. Shah A, Panjabi C. Allergic bronchopulmonary aspergillosis: a review of a disease with a worldwide distribution. J Asthma 2002;39:273-89. http://dx.doi.org/10.1081/JAS-120002284

3. Laennec RTH. A treatise on the diseases of the chest. New York: Hafner Publishing; 1962.

4. Salvi SS, Barnes PJ. Chronic obstructive pulmonary disease in non smokers. Lancet 2009;374:733-43. http://dx.doi.org/10.1016/50140-6736(09)61303-9

5. Murray CJ, Lopez AD. Alternative projections of mortality and disability by cause 1990-2020: Global Burden of Disease Study. Lancet 1997;349:1498-504. http://dx.doi.org/10.1016/S0140-6736(96)07492-2

6. Elias J. The relationship between asthma and COPD; lessons from transgenic mice. Chest 2004;126:111S-116S. http://dx.doi.org/10.1378/chest.126.2_suppl_1.111S

7. Agarwal R, Srinivas R, Jindal SK. Allergic bronchopulmonary aspergillosis complicating chronic obstructive pulmonary disease. Mycoses 2007;51:83-5.

8. Agarwal R, Hazarika B, Gupta D, Aggarwal AN, Chakrabarti A, Jindal SK. Aspergillus hypersensitivity in patients with chronic obstructive pulmonary disease: COPD as a risk factor for ABPA? Medical Mycology 2010;48:988-94. http://dx.doi.org/10.3109/13693781003743148

9. Global strategy for the diagnosis, management and prevention of chronic obstructive pulmonary disease. (Updated 2010). Accessed 25 March 2010 from: www.goldcopd.com

10. Chauhan B, Santiago L, Kirschmann DA, et al. The association of HLA DR alleles and $T$ cell activation with allergic bronchopulmonary aspergillosis. J Immunol 1997;159(8):4072-6.

11. Marchand E, Verellen-Dumoulin C, et al. Frequency of cystic fibrosis transmembrane conductance regulator gene mutations and $5 \mathrm{~T}$ allele in patients with allergic bronchopulmonary aspergillosis. Chest 2001;119:762-7.

http://dx.doi.org/10.1378/chest.119.3.762

12. Brouard J, Knauer N, Boelle PY, et al. Influence of interleukin-10 on Aspergillus fumigatus infection in patients with cystic fibrosis. J Infect Dis 2005;191:1988-91. http://dx.doi.org/10.1086/429964

13. Vaid M, Kaur S, Sambatakou H, Madan T, Denning DW, Sarma PU. Distinct alleles of mannose-binding lectin (MBL) and surfactant proteins A (SP-A) in patients with chronic cavitary pulmonary aspergillosis and allergic bronchopulmonary aspergillosis. Clin Chem Lab Med 2007;45:183-6. http://dx.doi.org/10.1515/CCLM.2007.033

14. Chauhan B, Santiago L, Hutcheson PS, et al. Evidence for the involvement of two different MHC class II regions in susceptibility or protection in allergic bronchopulmonary aspergillosis. J Allergy Clin Immunol 2000;106:723-9. http://dx.doi.org/10.1067/mai.2000.109913

15. Orie NGM, Sluiter HJ, de Vries K, et al. The host factor in bronchitis. In: Orie NGM Sluiter HJ, eds. Bronchitis. Assen, the Netherlands: Royal van Gorcum, 1961;43-59.

16. Taylor RJ, Joyce H, Gross E, Holland F, Pride NB. Bronchial reactivity to inhaled histamine and annual rate of decline in FEV1 in male smokers and ex-smokers. Thorax 1985;40:9-16. http://dx.doi.org/10.1136/thx.40.1.9

17. Burrows $B$, Halonen $M$, Barbee RA, Lebowitz MD. The relationship of serum immunoglobulin $\mathrm{E}$ to cigarette smoking. Am Rev Respir Dis 1981;124:523-5.

18. Taylor RG, Gross E, Joyce $\mathrm{H}$ et al. Smoking, allergy and the differential white blood cell count. Thorax 1985;40:17-22. http://dx.doi.org/10.1136/thx.40.1.17

19. Brightling $C E$, Monteiro $W$, Ward $R$ et al. Sputum eosinophilia and short-term response to prednisolone in chronic obstructive pulmonary disease: a randomized controlled trial. Lancet 2000;356:1480-5. http://dx.doi.org/10.1016/S0140-6736(00)02872-5

20. Chanez P, Vignola AM, O'Shaugnessy $T$, et al. Corticosteroid reversibility in COPD is related to features of asthma. Am J Respir Crit Care Med 1997;155:1529-34.

21. Burrows B, Hasan FM, Barbee RA, et al. Epidemiologic observations on eosinophilia and its relation to respiratory disorders. Am Rev Respir Dis 1980;122:709-19.

22. Dye JA, Adler KB. Effects of cigarette smoke on epithelial cells of the respiratory tract. Thorax 1994;49:825-34. http://dx.doi.org/10.1136/thx.49.8.825

23. Pepys J, Riddell RW, Citron KM, Clayton YM, Short El. Clinical and immunologic significance of Aspergillus fumigatus in the sputum. Am Rev Respir Dis 1959;80:16780.

24. Meersseman W, Vandecasteele SJ, Wilmer A, Verbeken E, Peetermans WE, Wijngaerden EV. Invasive aspergillosis in critically ill patients without malignancy. Am J Respir Crit Care Med 2004;170:621-5. http://dx.doi.org/10.1164/rccm.200401-0930C

25. Maurya V, Gugnani HC, Sarma PU, Madan T, Shah A. Sensitization to Aspergillus antigens and occurrence of allergic bronchopulmonary aspergillosis in patients with asthma. Chest 2005;127:1252-9. http://dx.doi.org/10.1378/chest.127.4.1252

26. Kumar A, Shah A. Occurrence of upper airway symptoms and their impact on quality of life in patients with chronic obstructive pulmonary disease. Respirology 2008;13 (Suppl 5):A109.

27. Shah A. Allergic bronchopulmonary aspergillosis: an Indian perspective. Curr Opin Pulm Med 2007;13:72-80. http://dx.doi.org/10.1097/M CP.0b013e328011a284

28. Chakrabarti A, Sethi S, Raman DS, Behera D. Eight-year study of allergic bronchopulmonary aspergillosis in an Indian teaching hospital. Mycoses 2002; 45:295-9. http://dx.doi.org/10.1046/j.1439-0507.2002.00738.x

29. Greenberger PA. Allergic bronchopulmonary aspergillosis. J Allergy Clin Immunol 2002;110:685-92.

30. Cerveri I, Dore R, Corsico A, et al. Assessment of emphysema in COPD: A functional and radiological study. Chest 2004;125:1714-18. http://dx.doi.org/10.1378/chest.125.5.1714

31. Madani A, Keyzer C, Gevenois PA. Quantitative computed tomography assessment of lung structure and function in pulmonary emphysema. Eur Respir J 2001;18:720-30. http://dx.doi.org/10.1183/09031936.01.00255701 